\title{
基于滚动引导滤波的红外与可见光图像融合算法
}

\author{
陈 峰, 李 敏, 马 乐, 邱晓华 \\ (高新技术研究所, 陕西 西安 710025)
}

\begin{abstract}
摘要: 针对红外与可见光图像融合时, 易产生细节丢失、噪声抑制不佳等问题, 本文提出了一种改进 的滚动引导滤波融合算法。该算法充分利用了滚动引导滤波边缘和局部亮度保持特性，在通过均值滤 波将输入图像分解为基础层与细节层的基础上, 结合滚动引导滤波与高斯滤波获取输入图像的显著 图, 利用不同尺度参数的引导滤波对显著图优化得到权重图, 将权重图作为权重分别指导基础层与细 节层的融合，最后联合融合后的子图重构得到融合图像。针对 3 类测试数据进行的融合实验表明，与 非下采样轮廓波变换、基于引导滤波、基于显著性检测的两个尺度的图像融合等经典方法相比，本文 方法得到的融合图像不但从主观视觉效果上细节信息更丰富、目标对比度加强, 并且在非线性相关信 息摘、相位一致性等 6 项客观评价指标上均具有较好的效果。
\end{abstract}

关键词: 滚动引导滤波; 显著图; 图像融合; 权重图

中图分类号：TN219 文献标识码：A 文章编号：1001-8891(2020)01-0054-08

\section{Infrared and Visible Image Fusion Algorithm Based on the Rolling Guidance Filter}

\author{
CHEN Feng, LI Min, MA Le, QIU Xiaohua \\ (High-tech Institute, Xi'an 710025, China)
}

\begin{abstract}
For the fusion of infrared and visible images, it is easy to produce problems such as missing detail information and suppressing less noise. In this paper, an improved fusion algorithm is proposed by applying the characteristics of a rolling guidance filter, which preserves edge and local brightness. First, the input images are decomposed into base and detail layers by mean filtering. Second, the saliency maps of the input images are obtained by combining the rolling guidance and Gaussian filters. The weight maps are then optimized by guided filters of different scales. The optimized maps are used to instruct the fusion of the base and detail layers. Finally, the fused image is reconstructed by combining the merged sub-images. The method of this paper is superior on the six indicators, such as nonlinear correlation information entropy and phase consistency, compared to the classical methods such as non-subsampled contourlet transform(NSCT), image fusion with guided filtering(GFF), and two-scale image fusion based on visual saliency(TSIFVS).
\end{abstract}

Key words: the rolling guidance filter(RGF), the saliency features, image fusion, edge preserving filter, weight map

\section{0 引言}

图像融合本质上是一种图像增强技术, 旨在通过 提取不同传感器 (如红外与可见光) 或同种传感器采 集的图像 (如多聚焦图像) 的互补信息进行融合, 生 成信息更丰富、图像特征更多的融合图像。红外与可
见光图像融合技术是当前图像融合领域研究的热点 之一。红外传感器通过捕捉目标的热辐射信息, 可生 成黑暗和雨雾等恶劣气候条件下的图像, 图像中红外 目标突出, 但空间分辨率较低、细节信息较少, 而场 景对应的可见光图像纹理、边缘等细节信息丰富, 分 辨率较高。故综合这两类图像的互补信息, 可生成一

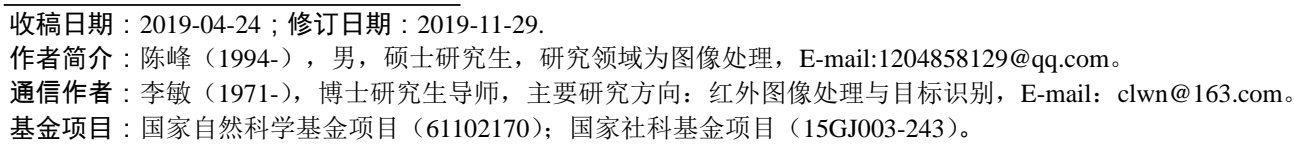


幅红外目标突出、纹理、边缘细节信息丰富的图像, 此类图像在检测跟踪、目标识别、监控等军事和民用 领域的应用更有优势。

根据采用的理论和工具的差异, 目前的图像融合 算法一般可分为基于多尺度变换、基于稀疏表示、基 于神经网络、基于子空间、基于显著性以及上述方法 结合的混合模式, 其中基于多尺度变换的方法是当前 该领域中研究最多的 ${ }^{[1-2]}$ 。最早用于图像融合中的理论 是基于金字塔变换和小波变换的图像融合算法 ${ }^{[3]}$,比如

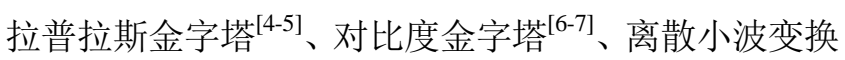
(discrete wavelet transformation, DWT) ${ }^{[8]}$ 等。针对离 散小波变换不具有平移不变性、方向选择有限的问题, 又有学者提出了融合性能更好的双树复小波变换 ${ }^{[9]}$ 。此 外, 方向灵敏度更高的轮廓波变换 (contourlet transform）、剪切波变换（shearlet transform）以及具 有平移不变性的非下采样轮廓波变换 （non-subsampled contourlet transform, NSCT)、非下 采样剪切波变换（nonsubsampled shearlet transform, NSST）等多尺度变换工具也相继被众多学者用于图 像融合领域, 2019 年 $\mathrm{Ma}$ 等人在文献[1]中对这些方法 进行了综述。NSCT 等多尺度变换方法首先将图像分 解为高频与低频系数, 然后采用不同的权重分别对高 低频系数进行组合, 再经逆变换得到融合图像。此类 方法的融合质量较高, 但时间消耗较大。

由于保边滤波器具有噪声平滑、边缘保留等特 性, 近年来, 众多学者开始将保边滤波成功运用于图 像融合中 ${ }^{[10-13]}$ 。2013 年 $\mathrm{Li}$ 等人提出一种基于引导滤 波的图像融合算法 ${ }^{[10]}$ (Image fusion with guided filtering, GFF）, 采用均值滤波对图像分解, 结合拉 普拉斯滤波和高斯滤波来获取显著图, 并首次将引导 滤波用于权重图的构造, 解决了初始权重图目标边缘 未对齐的问题。2019 年, Zhang 等人在文献[13]中设 计了一个梯度滤波器来获取显著图, 可提取更多的梯 度特征。同年, Ma 等人在文献[14]中提出用中值滤波 图像与均值滤波图像做差值, 取绝对值后, 再经高斯 滤波得到显著图, 该方法保留的细节特征较多, 但中 值滤波在去噪的同时也会去除一些高频信息。2014 年 Zhang 等人提出一种滚动引导滤波 (the Rolling Guidance Filter, RGF) 框架 ${ }^{[15]}$, 区别于双边滤波、引 导滤波, 该滤波器可以有效消除光晕, 在平滑小尺度 目标的同时恢复大尺度目标边缘, 2017 年 Ma 等人结 合滚动引导滤波与高斯滤波对源图像进行多尺度分 解 ${ }^{[12]}$ 。

在以上研究的基础上, 本文提出结合滚动引导滤 波与高斯滤波获取显著图, 再利用大、小尺度参数的
引导滤波对其优化分别得到基础层与细节层的权重 图, 利用权重图分别指导基础层与细节层的融合, 最 后联合融合的子图像, 得到融合图像。针对 3 种测试 数据, 将本文算法与 NSCT、GFF、基于多尺度变换 与稀疏表示 (LP and sparse representation, LPSR) 等 经典算法进行了实验对比分析, 验证了本文算法的优 越性。

\section{1 基于滚动引导滤波的显著图提取}

\section{1 滚动引导滤波}

滚动引导滤波是一种高效的尺度感知滤波器, 在 平滑小尺度目标及纹理细节时, 可以迭代恢复大尺度 目标的边缘。该滤波器的原理结构可细分为: 小尺度 结构平滑和大尺度结构边缘恢复。首先采用高斯滤波 器对源图像进行小尺度结构的平滑。设 $I$ 为输入图像, $J$ 为高斯模糊后的图像, 表示为:

$$
J=f_{1}\left(I, \sigma_{\mathrm{s}}\right)=\frac{1}{K_{p}} \sum_{q \in N(p)} \exp \left(-\frac{\|p-q\|^{2}}{2 \sigma_{\mathrm{s}}^{2}}\right) I(q)
$$

式中: $K_{p}=\sum_{q \in N(p)} \exp \left(-\frac{\|p-q\|^{2}}{2 \sigma_{\mathrm{s}}^{2}}\right)$ 用于将高斯滤波中 的所有权重归一化, $p 、 q$ 分别表示图像中不同像素点 的坐标; $I(q)$ 表示像素点 $q$ 的灰度值; $N(p)$ 为像素点 $p$ 的领域像素集合。标准差 $\sigma_{\mathrm{s}}$ 用于控制高斯滤波核的窗 口大小, 凡是像素距离小于 $\sigma_{\mathrm{s}}$ 的结构均会被平滑掉, 但同时大尺度结构边缘也会被一定程度的模糊掉。

滚动引导滤波的第二步是采用双边滤波 (bilateral filter, BF) ${ }^{[16]}$ 将第一步中高斯滤波的结果 $J$ 作为引导 图像, 初始输入图像 $I$ 作为输入图像, 迭代恢复被模 糊的大尺度结构边缘。下一次迭代的引导图像和输入 图像分别为上一次迭代的输出图像、初始的输入图像。 随着迭代次数的增加, 被模糊掉的大尺度结构边缘会 越来越清晰。据文献[15]所述, 此处的双边滤波可根据 实际应用需求采用引导滤波 (guided filter, GF) [17]、 域变换 (domain-transform filter, DTF) ${ }^{[18]}$ 等滤波器 替代, 本文采用双边滤波的方案, 表示为:

$$
\begin{aligned}
J^{t+1}(p) & =f_{2}\left(J^{t}, \sigma_{\mathrm{s}}, \sigma_{\mathrm{r}}\right) \\
& =\frac{1}{K_{p}} \sum_{q \in N(p)} \exp \left(-\frac{\|p-q\|^{2}}{2 \sigma_{\mathrm{s}}^{2}}-\frac{\left\|J^{t}(p)-J^{t}(q)\right\|^{2}}{2 \sigma_{\mathrm{r}}^{2}}\right) I(q)
\end{aligned}
$$

式中: $K_{p}=\sum_{q \in N(p)} \exp \left(-\frac{\|p-q\|^{2}}{2 \sigma_{\mathrm{s}}^{2}}-\frac{\left\|J^{t}(p)-J^{t}(q)\right\|^{2}}{2 \sigma_{\mathrm{r}}^{2}}\right)$ 用 于归一化, $I$ 与式(1)中的相同指初始输入图像, $J^{t}$ 表 
示上一次迭代输出图像, $t$ 指迭代的次数。当 $t=0$ 时, 设 $J^{0}$ 为常数, 式(2)退化为式(1); 当 $t=1$ 时, $J$ 表示 式(1)的输出图像。 $J^{t}(p)$ (或 $J^{t}(q)$ ) 指 $J^{t}$ 图像中坐标为 $p 、 q$ 的像素点的灰度值。 $\sigma_{\mathrm{r}}$ 用于控制强度差 (如灰度 差) 的权重。由式(2)可知, 滚动滤波器通过 $\sigma_{\mathrm{s}}$ 与 $\sigma_{\mathrm{r}}$ 分别控制滤波的空间范围权重与强度差范围权重。

设 $t=0$ 时, $J^{0}$ 为常数, 可得到滚动引导滤波的定 义式:

$$
O=f_{\mathrm{rgf}}\left(I, \sigma_{\mathrm{s}}, \sigma_{\mathrm{r}}, T\right)
$$

式中: $I$ 为输入图像; $O$ 为输出图像; $T$ 为总的迭代次 数。

\section{2 显著图提取方法}

自然图像由不同尺度的目标组成, 且包含了不同 的尺度结构 ${ }^{[15]}$, 图像的显著特征通常指图像中不同目 标的边缘、角等信息。

滚动引导滤波在迭代过程中通过 $\sigma_{\mathrm{s}}$ 和 $\sigma_{\mathrm{r}}$ 两参数控 制滤波的空间（距离）权重和范围（强度）权重, 可 实现对图像不同尺度的有效分割, 最终平滑小尺度结 构的同时可准确保留目标的边缘。将滚动引导滤波用 于平滑图像时, 在一定范围内, $\sigma_{\mathrm{s}}$ 值越大, 平滑掉的 细节越多; $\sigma_{\mathrm{r}}$ 值越大, 图像中保留的目标边缘也会趋 于模糊。依据这一特性, 可利用该滤波器提取图像的 高通图像, 即利用原图与经该滤波平滑后的图像做差 值并取绝对值。此时 $\sigma_{\mathrm{s}}$ 和 $\sigma_{\mathrm{r}}$ 取值越小, 高通图像保留 的细节信息丰富。由于该高通图像仍含有噪声, 需经 高斯滤波去噪, 得到噪声较少的显著图。结合滚动引 导滤波与高斯滤波提取显著图的表达式可表示为:

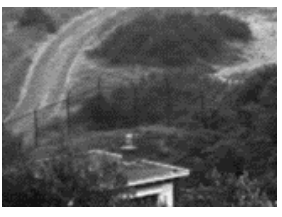

(a)Visible image

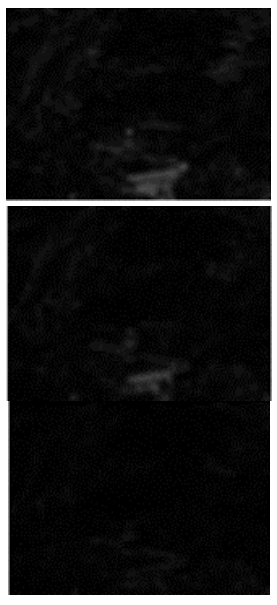

$\sigma_{\mathrm{r}}=0.05$ $\sigma_{\mathrm{r}}=0.005$

图 1 不同 $\sigma_{\mathrm{s}}$ 和 $\sigma_{\mathrm{r}}$ 下提取的显著图

$$
S=\left|I-f_{\text {rgf }}(I)\right|^{*} g\left(r_{\mathrm{g}}, \sigma_{\mathrm{g}}\right)
$$

式中: $S$ 表示提取的显著图; $f_{\mathrm{rgf}}(\cdot)$ 表示滚动引导滤波; $g$ 表示高斯滤波; $r_{\mathrm{g}}$ 和 $\sigma_{\mathrm{g}}$ 为高斯滤波核的窗口大小和 标准差, 均设置为 5 。为进一步分析 $\sigma_{\mathrm{s}}$ 和 $\sigma_{\mathrm{r}}$ 两参数对 提取的显著图的影响, 下面分别对一组经严格配准后 的红外与可见图像进行实验分析, 实验图像如图 1(a)、 图 1 (b)所示。

在图 1 中, 左边 3 列是对可见光图像提取的显著 图。由图可知, 当取定 $\sigma_{\mathrm{s}}$ (或者 $\sigma_{\mathrm{r}}$ ) 时, 在一定范围 内, $\sigma_{\mathrm{r}}\left(\right.$ 或 $\left.\sigma_{\mathrm{s}}\right)$ 取值越大, 提取的显著图包含的细节 信息越丰富，当 $\sigma_{\mathrm{s}}$ 和 $\sigma_{\mathrm{r}}$ 分别取 $16 、 0.5$ 时，提取的细 节信息最多，即右边三列最右上角的图像。图 1 中最 右边两列为 $\sigma_{\mathrm{s}}$ 取不同值、 $\sigma_{\mathrm{r}}$ 取 0.05 和 0.5 时, 当 $\sigma_{\mathrm{s}}$ 和 $\sigma_{\mathrm{r}}$ 分别取 16、0.5 时提取的显著图包含的红外目标特 征最多, 轮廓最清晰, 即右边两列最右上角的图像。 综上所述, 当 $\sigma_{\mathrm{s}}$ 与 $\sigma_{\mathrm{r}}$ 分别取 16 与 0.5 时, 所提方法对 红外与可见光图像提取的显著图包含的细节信息最 丰富。

\section{2 基于滚动引导滤波的图像融合}

在文献[10]中提出将引导滤波用于权重图优化的 框架, 并基于均值滤波提出了一种融合方法, 本节首 先从融合框架、权重图构造方法进行介绍, 然后对本 文的权重图构造方法详细论述。由于均值滤波分解图 像效率较高, 文中仍采用均值滤波将图像分解为基础 层与细节层。

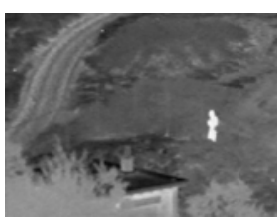

(b)Infrared image
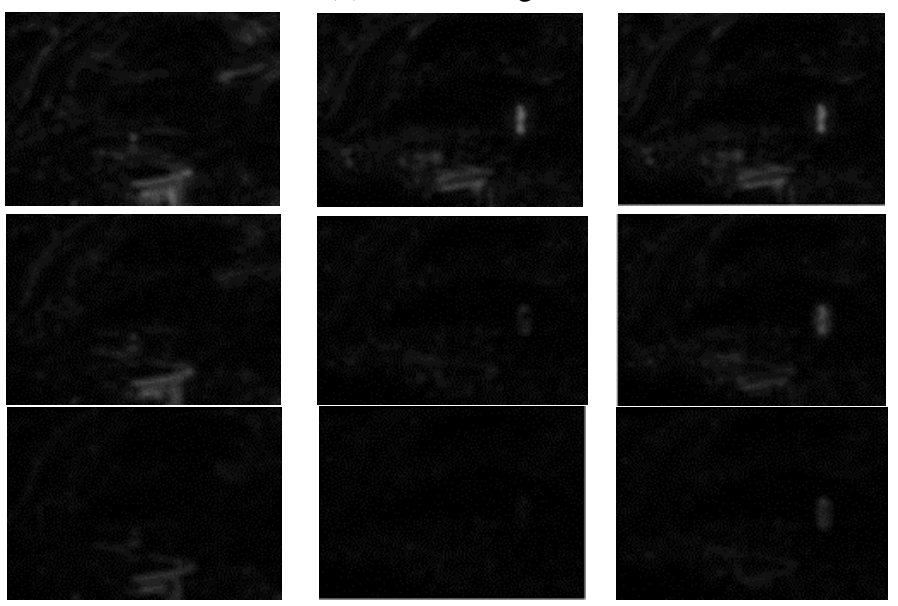

$\sigma_{\mathrm{r}}=0.5$

$\sigma_{\mathrm{r}}=0.05$

$\sigma_{\mathrm{r}}=0.5$

Fig 1 The saliency images extracted under different $\sigma_{\mathrm{s}}$ and $\sigma_{\mathrm{r}}$ 


\section{1 融合框架}

设 $I_{n}(n$ 的值为 $1 、 2)$ 为输入源图像, 通过均值 滤波将源图像分解为对应的基础层 $B_{n}$ 和细节层 $D_{n}$, 分解函数表示为:

$$
\left[\frac{B_{n}}{D_{n}}\right]=\left[\frac{I_{n} * f_{\text {ave }}}{I_{n}-I_{n} * f_{\text {ave }}}\right]
$$

式中: $f_{\text {ave }}$ 为均值滤波; “*”为滤波运算, 源图像经均 值滤波后可得到包含大量低频信息的基础层, 表示图 像的整体对比度和尺度较大的目标边缘。

设 $W_{n}{ }^{B} 、 W_{n}{ }^{D}(n$ 的值为 $1 、 2$ ) 分别为基础层与 细节层的融合系数, $\bar{B} 、 \bar{D}$ 分别为融合后的基础层与 细节层, 对融合后的基础层与细节层分别赋予权重为 1 的系数可重构得到融合图像，表示：

$$
\left[\frac{\bar{B}}{\bar{D}}\right]=\left[\frac{\sum_{n=1}^{N} W_{n}^{B} B_{n}}{\sum_{n=1}^{N} W_{n}^{D} D_{n}}\right]
$$

设 $F$ 为融合的结果图像, 分别对式(6)融合后的基 础层与细节层融合图像赋予值为 1 的权重, 重构得到 最终的融合图像, 表示为:

$$
F=\bar{B}+\bar{D}
$$

\section{2 权重图构造方法}

权重图的构造过程包括显著图、初级权重图、最 终权重图构造 3 个步骤。文献[10]利用了一个 $3 \times 3$ 的 拉普拉斯滤波和高斯滤波来构造显著图, 其中拉普拉 斯滤波可以增强图像的特征, 但同时也会增强噪声, 高斯滤波用于对图像去噪得到显著图, 可表示为:

$$
\left[\frac{S_{1}}{S_{2}}\right]=\left[\frac{\left|I_{1} * l\right| * g\left(r_{\mathrm{g}}, \sigma_{\mathrm{g}}\right)}{\left.\left|I_{2} * l\right| * g\left(r_{\mathrm{g}}, \sigma_{\mathrm{g}}\right)\right)}\right]
$$

式中: $l$ 为拉普拉斯滤波器; $g$ 为高斯滤波器; “*”为 滤波运算; $S_{1} 、 S_{2}$ 为提取的显著图。

通过比较显著图 $S_{1} 、 S_{2}$ 中相应像素点的显著值,

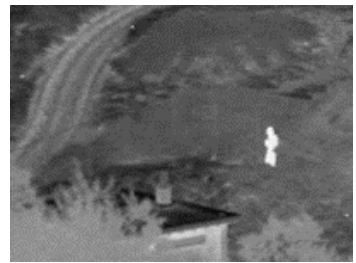

(a) Infrared image

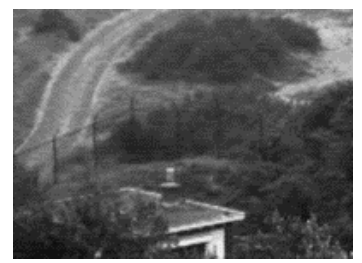

(b) Visible image

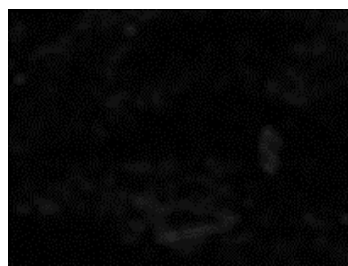

(a1) LP-G

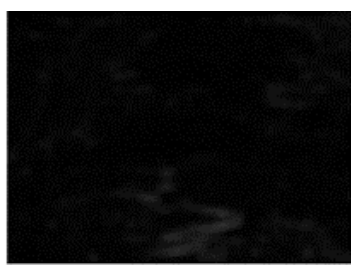

(b1) LP-G

得到初级权重图 (二进制图) $P_{1}$ 与 $P_{2}$, 表示为:

$$
P_{1}= \begin{cases}1, & S_{1}\left(x_{i}, y_{i}\right) \geq S_{2}\left(x_{i}, y_{i}\right) \\ 0, & S_{1}\left(x_{i}, y_{i}\right)<S_{2}\left(x_{i}, y_{i}\right)\end{cases}
$$

或者:

$$
\begin{aligned}
& P_{1}= \begin{cases}1, & S_{1}\left(x_{i}, y_{i}\right) \leq S_{2}\left(x_{i}, y_{i}\right) \\
0, & S_{1}\left(x_{i}, y_{i}\right)>S_{2}\left(x_{i}, y_{i}\right)\end{cases} \\
& P_{2}=1-P_{1}
\end{aligned}
$$

式中: $S_{1}\left(x_{i}, y_{i}\right) 、 S_{2}\left(x_{i}, y_{i}\right)$ 分别为各显著图中像素点 $\left(x_{i}\right.$, $\left.y_{i}\right)$ 的灰度值。

由于初级权重图中仍包含噪声, 且有的目标边缘 未对齐, 直接作为权重图融合, 可能会产生伪影, 故 采用引导滤波对其进行优化。分别以源图像 $\left(I_{1} 、 I_{2}\right)$ 为引导图像, 初级权重图为输入图像, 经引导滤波优 化后可获得最终的权重图。表示为:

$$
\left[\frac{W_{n}^{B}}{W_{n}^{D}}\right]=\left[\frac{G\left(P_{n}, I_{n}, r_{1}, \varepsilon_{1}\right)}{G\left(P_{n}, I_{n}, r_{2}, \varepsilon_{2}\right)}\right]
$$

\section{3 改进的权重图构造方法}

上述融合方法中采用拉普拉斯滤波器获取源图 像的显著特征图，拉普拉斯滤波的原理是利用 $3 \times 3$ 模板遍历整个输入图像, 取模板内所有像素点的平均 值代替中间像素点, 但加权平均的方式会导致获取的 边缘、纹理等高频信息不充分, 最终的融合图像细节 信息保留不够充分。

在上文中, 论证了将滚动引导滤波用于构造显著 图的可行性。结合式(4)与式(8)后可得新的显著图构造 算法，如下所示:

$$
\left[\frac{S_{1}^{\prime}}{S_{2}^{\prime}}\right]=\left[\frac{\left|I_{1}-f_{\mathrm{rgf}}\left(I_{1}\right)\right| * g\left(r_{\mathrm{g}}, \sigma_{\mathrm{g}}\right)}{\left|I_{2}-f_{\mathrm{rgf}}\left(I_{2}\right)\right| * g\left(r_{\mathrm{g}}, \sigma_{\mathrm{g}}\right)}\right]
$$

式中: $S_{1}{ }^{\prime} 、 S_{2}{ }^{\prime}$ 分别为经滚动引导滤波和高斯滤波后获 得的显著图。

图 2 是分别采用拉普拉斯滤波与高斯滤波 (LP-G)、

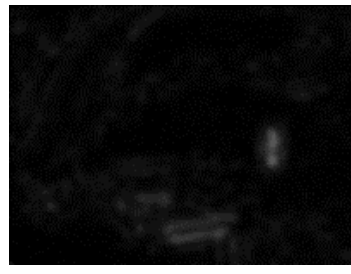

(a2) ME-AV-G

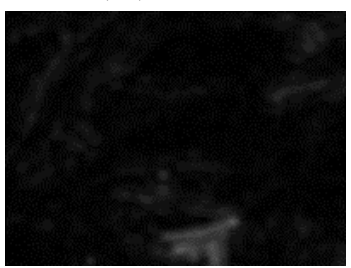

(b2) ME-AV-G

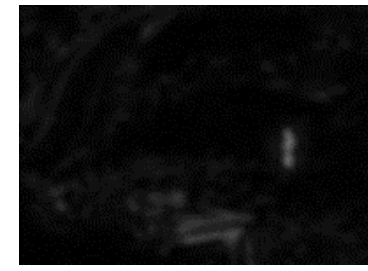

(a3) Ours

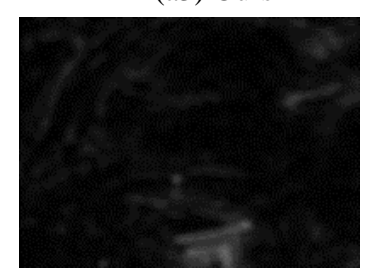

(b3) Ours

图 2 不同方法获取的显著图

Fig.2 The saliency images obtained by different methods 
中值滤波-均值滤波与高斯滤波（ME-AV-G）以及本 文方法对红外与可见光图像获得的显著图。比较红外 显著图可知, 本文方法得到的显著图中目标的轮廓更 清晰, 其他方法保留的红外目标较模糊; 比较可见光 显著图可知，本文方法保留的细节信息更丰富。

\section{4 融合算法}

基于滚动引导滤波的图像融合具体步骤如下所 示:

Step1: 利用均值滤波将红外与可见光图像分别分 解为基础层与细节层 $\left\{B_{n} 、 D_{n}\right\}$;

Step2: 利用滚动引导滤波与高斯滤波获得源图像 的显著图 $\left\{S_{1} 、 S_{2}\right\}$;

Step3: 比较显著图中的显著值, 获得初始权重图 $\left\{P_{1} 、 P_{2}\right\}$;

Step4: 利用不同尺度的引导滤波优化初始权重图 得到基础层与细节层的权重图 $\left\{W_{n}{ }^{B} 、 W_{n}{ }^{D}\right\}$;

Step5: 将得到的权重图作为权重系数分别指导基 础层与细节层的融合;

Step6: 将融合后的基础层与细节层分别以 1 为权 重系数, 重构得到最终的融合图像

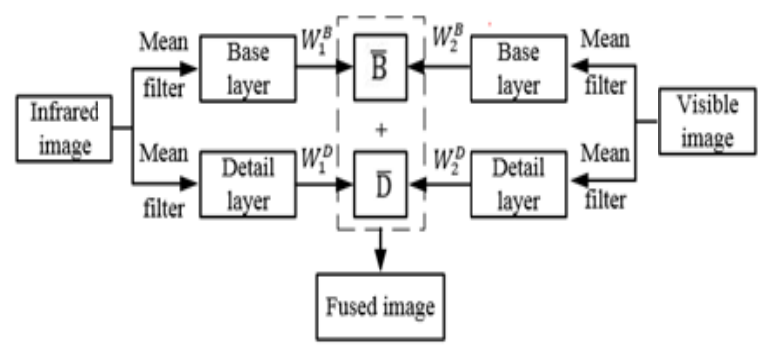

（a）融合框架图（a) Fusion framework

图 3 红外与可见光图像融合流程图
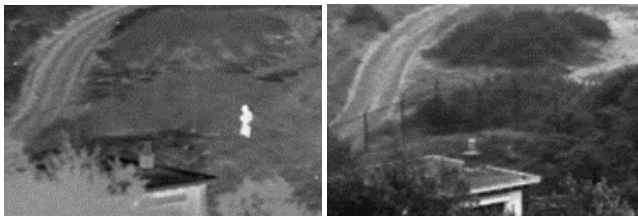

(a) "UN Camp"
图 3 中, 图(a)为采用均值滤波对图像单尺度分解 的融合框架图, 图(b)为源图像经滚动引导滤波、高斯 滤波、引导滤波等运算的基础层与细节层权重图构造 流程图。

\section{3 实验结果与分析}

\section{1 实验条件}

为进一步验证本文方法的有效性, 选择了 3 类典 型背景目标进行实验, 所选择的实验数据均为公开且 经严格配准后的图像。如图 4 所示。实验的运行环境 为: Intel i5, $2.3 \mathrm{GHz}$, 内存 $4 \mathrm{~GB}, 64$ 位 Windows7 操作系统, Matlab2017a。此外, 将本文方法与 $\mathrm{DWT}^{[8]}$

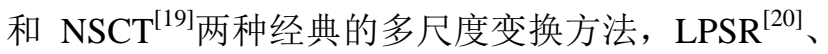
基于显著性检测的两个尺度的图像融合方法 ${ }^{[21]}$ (two-scale image fusion based on visual saliency, TSIFVS）两种最新提出的方法, 以及文献[10]所提出 的 GFF 方法 (这 5 种方法进行比较, 各方法的参数设 置均与原文献中保持一致, 本文方法的尺度参数 $\sigma_{\mathrm{s}}$ 和 $\sigma_{\mathrm{r}}$ 分别设置为 16 和 0.5 , 迭代次数采用文献[15]中的 最佳次数 4 。

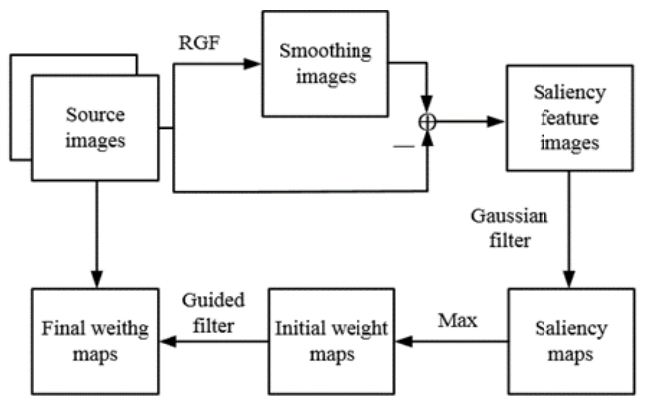

（b) 权重图构造流程图 (b) Weight map construction flow chart

Fig.3 Flow chart of infrared and visible image fusion

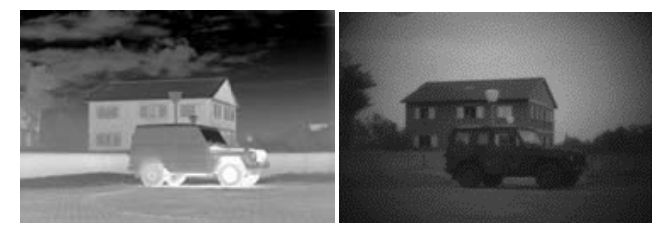

(b) "Marne"
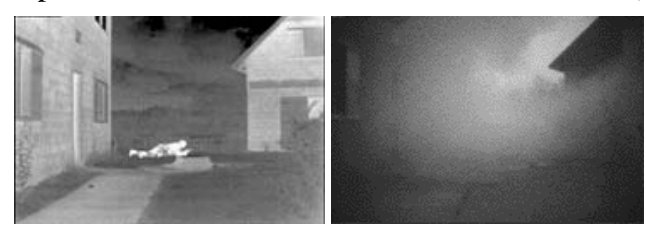

(c) "Smog environment"

图 43 对红外与可见光图像 Fig.4 Three pairs of infrared and visible images 


\section{2 实验结果分析}

图 5 为不同方法对测试数据 “UN Camp” 的融合 结果, 对比分析融合结果, DWT (图 5(c))、NSCT (图 5(d))两种传统的多尺度变换方法得到的融合图像, 目标不够突出, 整体亮度偏暗; GFF 方法（图 5(e)）, 目标附近植被和远处山丘纹理不清晰, 表示对红外噪 声抑制不充分; TSIFVS 方法 (图 5(f)), 目标较突出, 且目标周围的栅栏也较清晰, 但屋顶背景较暗, 目标 右边的植被较模糊, 表明融合了较多的红外信息; LPSR 方法（图 5(g)）, 目标较突出, 但目标右边的植 被不清晰; 本文方法（图 5(h)），目标突出，且目标 周围的栅栏、屋顶及远近位置的植被等背景都较清晰。

图 6 为不同方法在测试数据 “Marne” 上的融合 结果, 汽车为带内热源的目标, 相比于图 5 中人这一 小目标, 汽车的红外与可见光特征分布更复杂。观察 房子、车辆、天空、地面等目标背景, 图 6(c)、(d)两 种经典的方法融合的图像亮度信息较少, 含有的噪声

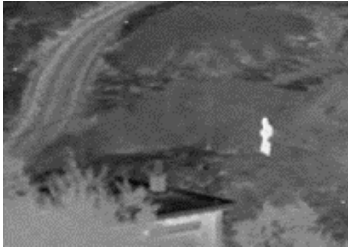

(a) Infrared image

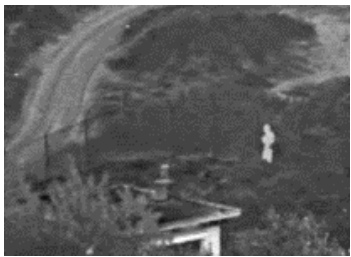

(e) GFF

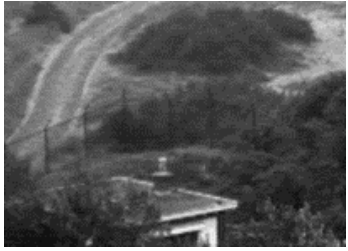

(b) Visible image



(f) TSIFVS

较多，整体视觉效果较差。图 6(e)方法融合的可见光 细节信息较多, 但目标的红外特征较少且融合图像中 间较亮，周围较暗，特征融合存在不衔接的缺点。图 6(f)方法融合的红外信息较少，图 6(g)车辆融合特征 较明显, 但车窗处的细节信息较少一些, 房子整体外 观，有的地方较亮，有的偏暗。本文的方法（图 6(h)） 车辆红外特征较明显, 房子外观整体较亮, 融合效果 较好。

图 7 是针对测试数据 “Smog environment” 进行 的融合, 比较不同方法的融合结果可得出：(c)、(d) 两种方法融合的结果包含的噪声较多, 整体偏暗, 目 标不突出; (e)方法融合了较多的烟雾信息, 红外特征 融合较少; (f)方法, 目标突出, 细节信息丰富，但整 体偏暗; (g)方法融合的结果, 目标突出, 背景细节信 息丰富; (h)方法融合结果, 目标突出、轮廓清晰，房 子间的天空融合自然，具有与(g)方法相当的视觉效 果。

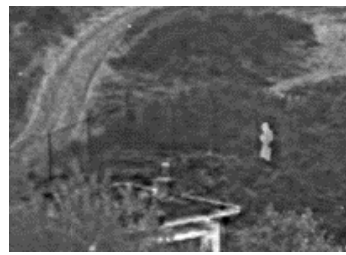

(c) DWT

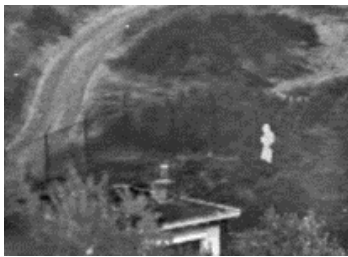

(g) LPSR

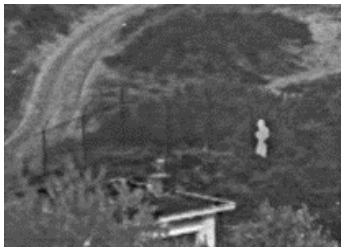

(d) NSCT

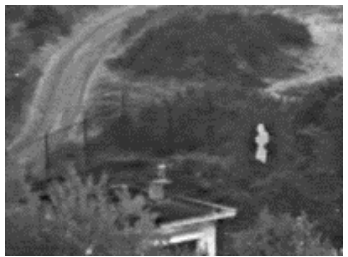

(h) Proposed

图 5 不同方法在测试数据 “UN Camp” 上的融合结果

Fig.5 Fusion results of different methods on the test data "UN Camp"

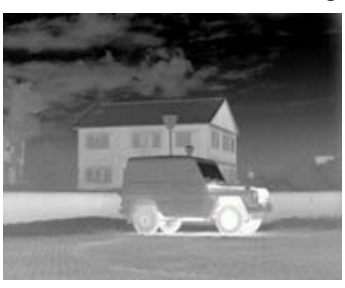

(a) Infrared image

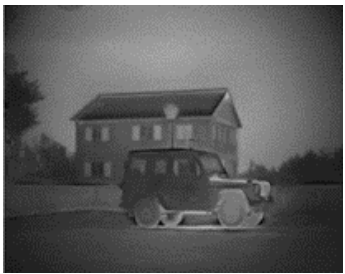

(e) GFF

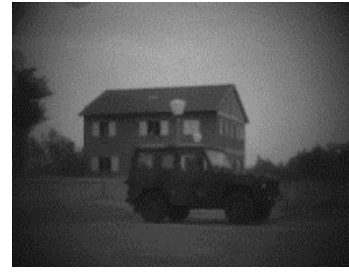

(b) Visible image

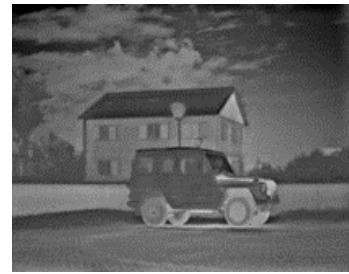

(f) TSIFVS

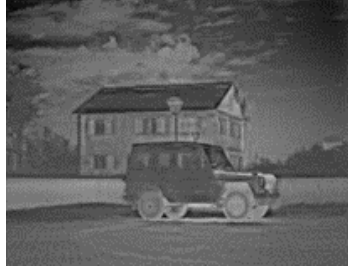

(c) DWT



(g) LPSR

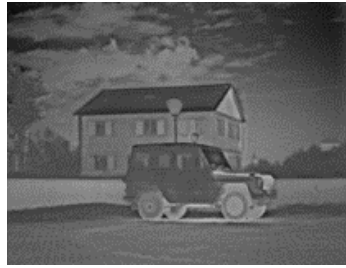

(d) NSCT

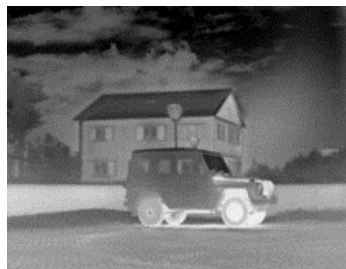

(h) Proposed

图 6 不同方法在测试数据 “Marne” 上的融合结果

Fig.6 Fusion results of different methods on the test data "Marne" 


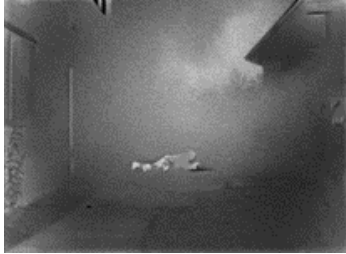

(a) Infrared image

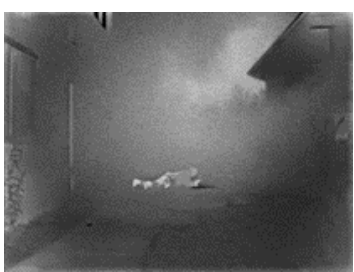

(e) GFF

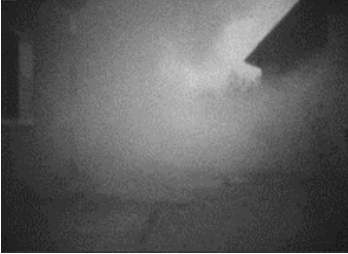

(b)Visible image

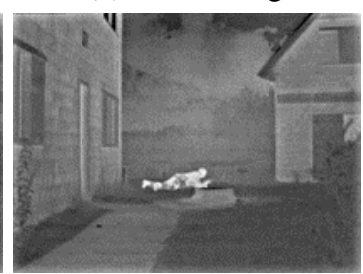

(f) TSIFVS

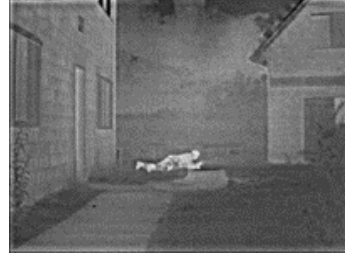

(c) DWT

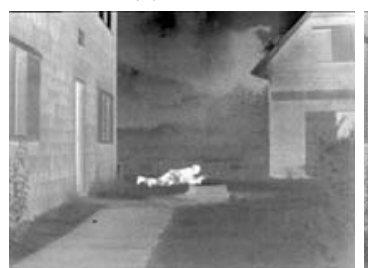

(g) LPSR

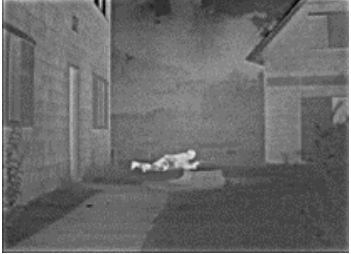

(d) NSCT

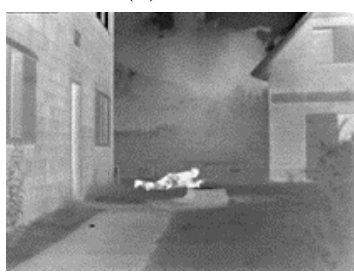

(h) Proposed

图 7 不同方法在测试数据 “Smog environment” 上的融合结果

Fig.7 Fusion results of different methods in the test data "smog environment"

\section{3 客观指标评价}

仅通过视觉效果来评价算法的融合效果, 评价结 果带有一定的主观性, 为更加客观地评价本文方法的 有效性, 还需利用客观评价指标进行评价。在文献[1] 与文献[22]中对一些经典及其衍生的客观评价指标进 行了实验分析与验证, 本文选择其中的 6 项指标: 基 于信息论的评价指标, 标准化互信息 $\left(Q_{\mathrm{NMI}}\right)$ 和非线 性相关信息熵（ $Q_{\mathrm{NCIE}}$ ）; 基于图像特征的评价指标, 特征互信息 $\left(Q_{\mathrm{FMI}}\right)$ 和相位一致性 $\left(Q_{\mathrm{P}}\right)$; 基于结构 相似度的评价指标, 局部结构相似度 $\left(Q_{\mathrm{Y}}\right)$; 基于人 类视觉感知的评价指标 $\left(Q_{\mathrm{CB}}\right)$ 。

标准化互信息表示从输入图像转换到融合图像 中的信息量; 非线性相关信息熵表示融合图像与输入 图像之间的非线性相关程度; 特征互信息从边缘、细 节、对比度这 3 个方面综合评价从输入图像到融合图 像转换的特征信息; 相位一致性表示融合图像包含的 边缘、角度信息; 局部结构相似度表示融合图像与输 入图像的局部结构相似程度; 基于人类视觉感知的评 价 $\left(Q_{\mathrm{CB}}\right)$ 表示融合图像获得的对比度信息。

从表 1、2 可以发现经本文改进的方法在非线性 相关信息熵 $\left(Q_{\mathrm{NCIE}}\right)$ 、标准化互信息 $\left(Q_{\mathrm{NMI}}\right)$ 、特征 互信息 $\left(Q_{\mathrm{FMI}}\right)$ 、相位一致性 $\left(Q_{\mathrm{P}}\right)$ 、局部结构相似 度 $\left(Q_{\mathrm{Y}}\right)$ 、人类视觉感知 $\left(Q_{\mathrm{CB}}\right)$ 这 6 个评价指标方 面都有较大幅度的提升。在表 1 中, 本文方法的 $Q_{\mathrm{CB}}$ 数值比 NSCT 算法的低一些, 但其他的指标综合评价 均高于 NSCT 算法。在表 3 中, 本文方法的 $Q_{\mathrm{FMI}}$ 、 $Q_{P} 、 Q_{\mathrm{Y}} 、 Q_{\mathrm{CB}}$ 客观评价指标均高于其它方法, $Q_{\mathrm{NCIE}}$ 比 GFF 方法的值略低, 但 $Q_{\mathrm{NMI}}$ 这一基于信息摘的评 价指标比 GFF 方法高; 本文的 $Q_{\mathrm{NMI}}$ 值低于 LPSR 方 法, 但 $Q_{\mathrm{NCIE}}$ 值比其高。
表 1 测试数据 “UN Camp” 融合结果客观指标比较

Table 1 Comparison of objective indicators oftest data "UN Camp" fusion results

\begin{tabular}{lllllll}
\hline Metrics & DWT & NSCT & GFF & TSIFVS & LPSR & Proposed \\
\hline$Q_{\text {NCIE }}$ & 0.8031 & 0.8033 & 0.8033 & 0.8033 & 0.8034 & 0.8041 \\
$Q_{\text {NMI }}$ & 0.2042 & 0.2217 & 0.2289 & 0.2243 & 0.2229 & 0.2714 \\
$Q_{\text {FMI }}$ & 0.3660 & 0.4382 & 0.4691 & 0.3793 & 0.4272 & 0.4715 \\
$Q_{P}$ & 0.1975 & 0.3108 & 0.3971 & 0.2524 & 0.3206 & 0.4135 \\
$Q_{\mathrm{Y}}$ & 0.7351 & 0.8213 & 0.8462 & 0.7846 & 0.8201 & 0.8651 \\
$Q_{\mathrm{CB}}$ & 0.5386 & 0.5799 & 0.5384 & 0.5602 & 0.5732 & 0.5725 \\
\hline
\end{tabular}

表 2 测试数据 “Marne” 融合结果客观指标比较

Table 2 Comparison of objective indicators oftest data "Marne" fusion results

\begin{tabular}{lllllll}
\hline Metrics & DWT & NSCT & GFF & TSIFVS & LPSR & Proposed \\
\hline$Q_{\mathrm{NCIE}}$ & 0.8035 & 0.8037 & 0.8102 & 0.8037 & 0.8076 & 0.8077 \\
$Q_{\mathrm{NMI}}$ & 0.1780 & 0.2075 & 0.4880 & 0.2030 & 0.3704 & 0.3858 \\
$Q_{\mathrm{FMI}}$ & 0.3783 & 0.4232 & 0.5067 & 0.3905 & 0.4350 & 0.4802 \\
$Q_{P}$ & 0.2805 & 0.3924 & 0.2809 & 0.4436 & 0.3651 & 0.5962 \\
$Q_{\mathrm{Y}}$ & 0.6846 & 0.7487 & 0.9056 & 0.7094 & 0.7269 & 0.8569 \\
$Q_{\mathrm{CB}}$ & 0.4367 & 0.4507 & 0.3589 & 0.4393 & 0.4979 & 0.5083 \\
\hline
\end{tabular}

表 3 测试数据 “Smog environment” 融合结果客观指标比较

Table 3 Comparison of objective indicators for the fusion of test data "Smog environment "

\begin{tabular}{lllllll}
\hline Metrics & DWT & NSCT & GFF & TSIFVS & LPSR & Proposed \\
\hline$Q_{\text {NCIE }}$ & 0.8031 & 0.8032 & 0.8093 & 0.8033 & 0.8124 & 0.8089 \\
$Q_{\text {NMI }}$ & 0.1931 & 0.2093 & 0.4832 & 0.2125 & 0.5273 & 0.4602 \\
$Q_{\text {FMI }}$ & 0.3736 & 0.4183 & 0.4993 & 0.4104 & 0.4315 & 0.4997 \\
$Q_{P}$ & 0.2776 & 0.3865 & 0.3523 & 0.4570 & 0.3524 & 0.6158 \\
$Q_{\mathrm{Y}}$ & 0.7193 & 0.7831 & 0.8849 & 0.7971 & 0.7971 & 0.9282 \\
$Q_{\mathrm{CB}}$ & 0.5390 & 0.5588 & 0.4872 & 0.5615 & 0.5949 & 0.6040 \\
\hline
\end{tabular}




\section{4 总结}

本文利用滚动引导滤波在边缘和局部亮度保持 的特性, 结合高斯滤波去噪的特性来获取显著图, 利 用不同尺度的引导滤波对其优化, 得到基础层与细节 层的权重图, 利用权重图指导基础层与细节层的融 合, 联合融合后的子图像重构得到融合图像。文中分 析了滚动引导滤波的尺度参数 $\sigma_{\mathrm{s}}$ 和 $\sigma_{\mathrm{r}}$ 对提取的显著图 的影响。通过与 LP-G 方法、ME-AV-G 方法提取的显 著图比较, 结合滚动引导滤波与高斯滤波的方法提取 的显著图边缘、亮度等细节特征更多。最后针对 3 种 不同的测试数据进行实验, 结果表明: 本文方法较好 地保留了红外与可见光图像中的目标背景等信息, 与 NSCT、GFF、TSIFVS 等方法相比较, 融合图像在主 观视觉分析和客观评价指标上均更有优势, 是一种有 效的融合方法。但本文方法仅对图像进行了两个尺度 的分解, 下一步将对更多尺度级的分解与融合进行研 究。

\section{参考文献:}

[1] MA J, MA Y, LI C. Infrared and visible image fusion methods and applications: A survey[J]. Information Fusion, 2019, 45:153-178.

[2] XIN J, QIAN J, YAO S, et al. A Survey of Infrared and Visual Image Fusion Methods[J]. Infrared Physics \& Technology, 2017, 85: 478-501.

[3] 封子军. 像素级图像融合算法研究[D]. 成都: 电子科技大学, 2014. FENG Z J. Research on pixel-level image fusion method[D]. Chengdu: University of Electronic Science and Technology, 2014.

[4] YU X, REN J, CHEN Q, et al. A false color image fusion method based on multi-resolution color transfer in normalization YCBCR space[J]. Optik-International Journal for Light and Electron Optics, 2014, 125(20): 6010-6016.

[5] Vanmali A V, Gadre V M. Visible and nir image fusion using weightmap-guided Laplacian-Gaussian pyramid for improving scene visibility[J]. Sādhanā, 2017, 42(7): 1063-1082.

[6] JIN H, XI Q, WANG Y, et al. Fusion of visible and infrared images using multi-objective evolutionary algorithm based on decomposition[J]. Infrared Phys. Technol., 2015, 71: 151-158.

[7] XU H, WANG Y, WU Y, et al. Infrared and multi-type images fusion algorithm based on contrast pyramid transform[J]. Infrared Phys. Technol., 2016, 78: 133-146.
[8] Chipman L J, Orr T M, Graham L N. Wavelets and image fusion[C]//International Conference on Image Processing. 1995: 248-251.

[9] ZUO Y, LIU J, BAI G, et al. Airborne infrared and visible image fusion combined with region segmentation[J]. Sensors, 2017, 17(5): 1127.

[10] LI S, KANG X, HU J. Image Fusion With Guided Filtering[J]. IEEE Transactions on Image Processing, 2013, 22(7): 2864-2875.

[11] ZHU J, JIN W, LI L, et al. Multiscale infrared and visible image fusion using gradient domain guided image filtering[J]. Infrared Physics \& Technology, 2017: S1350449517305042.

[12] MA J, ZHOU Z, WANG B, et al. Infrared and visible image fusion based on visual saliency map and weighted least square optimization[J]. Infrared Physics \& Technology, 2017, 82: 8-17.

[13] ZHANG Y, WEI W, YUAN Y. Multi-focus image fusion with alternating guided filtering[J]. Signal Image and Video Processing, 2019, 13(4): 727-735.

[14] MA T, JIE M, BIN F, et al. Multi-scale decompotion based fusion of infrared and visible image via total variation and saliency analysis[J]. Infrared Physics \& Technology, 2018, 92: 154-162.

[15] ZHANG Q, SHEN X, XU L, et al. Rolling Guidance Filter[C]// European Conference on Computer Vision, 2014: 815-830.

[16] Tomasi C, Manduchi R. Bilateral Filtering for Gray and Color Images[J]. Iccv, 1998: 839-846

[17] HE K, SUN J, TANG X. Guided Image Filtering[J]. IEEE Transactions on Pattern Analysis \& Machine Intelligence, 2013, 35(6): 1397-1409.

[18] Gastal E S L, Oliveira M M . Domain transform for edge-aware image and video processing[J]. ACM Transactions on Graphics, 2011, 30(4): 1.

[19] Adu J, GAN J, WANG Y, et al. Image fusion based on nonsubsampled contourlet transform for infrared and visible light image[J]. Infrared Physics \& Technology, 2013, 61: 94-100.

[20] LIU Y, LIU S, WANG Z. A general framework for image fusion based on multi-scale transform and sparse representation[J]. Information Fusion, 2015, 24: 147-164.

[21] Bavirisetti D P, Dhuli R. Two-scale image fusion of visible and infrared images using saliency detection[J]. Infrared Physics \& Technology, 2016, 76: 52-64.

[22] LIU Z, Blasch E, XUE Z, et al. Objective Assessment of Multiresolution Image Fusion Algorithms for Context Enhancement in Night Vision: A Comparative Study[J]. IEEE Transactions on Pattern Analysis \& Machine Intelligence, 2011, 34(1): 94-109. 